\title{
Gender Classification Using Interlaced Derivative Patterns
}

\author{
Ameneh Shobeirinejad \\ School of Engineering \\ Griffith University \\ Brisbane, Queensland, Australia \\ a.shobeirinejad@griffith.edu.au
}

\author{
Yongsheng Gao \\ School of Engineering, Griffith University \\ Queensland Research Lab, National ICT Australia \\ Brisbane, Queensland, Australia \\ yongsheng.gao@\{griffith.edu.au,nicta.com.au\}
}

\begin{abstract}
Automated gender recognition has become an interesting and challenging research problem in recent years with its potential applications in security industry and humancomputer interaction systems. In this paper we present a novel feature representation, namely Interlaced Derivative Patterns (IDP), which is a derivative-based technique to extract discriminative facial features for gender classification. The proposed technique operates on a neighborhood around a pixel and concatenates the extracted regional feature distributions to form a feature vector. The experimental results demonstrate the effectiveness of the IDP method for gender classification, showing that the proposed approach achieves $29.6 \%$ relative error reduction compared to Local Binary Patterns (LBP), while it performs over four times faster than Local Derivative Patterns (LDP).
\end{abstract}

Keywords—gender recognition; performance evaluation; local derivative pattern; Interlaced Derivative Pattern

\section{INTRODUCTION}

Automatic face recognition and analysis is a challenging field in computer vision which allows many interesting applications in security industry and psychology. Examples include face detection, person identification, age estimation and gender classification. Gender classification can significantly improve human identification as it reduces the process of matching the face in the databases. This is also useful in many applications that may be more specific to a particular gender.

Gender classification methods can be roughly divided into appearance-based and feature-based methods [7]. The very early research on automatic gender classification goes back to the beginning of the 1990s. The first attempts were reported by Cottrell and Metcalfe [3] and Golomb et al. [4]. Each of them used a multi-layer neural network approach to identify gender from face images. Gutta et al. [5] presented a hybrid approach consisting of radial basis function networks and inductive decision trees. Moghaddam et al. [8] experimented with a support vector machine (SVM) and radial basis function (RBF) kernel.

The abovementioned techniques are appearance-based methods; they identify gender by training images without extracting any geometrical features. Brunelli and Poggio [2] used HyperBF networks to extract a set of 16 geometric features from frontal face images. Sun et al. [12] proposed that feature selection is an important issue for gender classification and applied genetic algorithms to select a subset of features from frontal images. Saatci and Town [11] used an SVM that was trained with the features extracted by an active appearance model (AAM). Lian and $\mathrm{Lu}$ [6] experimented with local binary pattern (LBP) and SVM, and achieved considerably high results.

The LBP features were originally designed for texture description [9]. The technique has been successfully implemented in other applications such as face recognition [1] and gender classification [6]. Recently a powerful operator, called Local Derivative Pattern (LDP) [13], has surpassed LBP in face recognition tasks. LDP encodes directional pattern features based on local derivative variations, while LBP can conceptually be considered as a nondirectional first-order local pattern. The high-order LDP captures more detailed discriminative information which exceeds the LBP features in terms of face recognition precision.

In this paper, we investigate the feasibility and effectiveness of LDP on gender classification and propose a novel representation of facial images, the Interlaced Derivative Pattern (IDP), for gender recognition. We discuss that by applying more effective techniques to extract features for gender identification, higher rates in gender classification will be achieved. The proposed method is evaluated on FRGC ver.2.0 database [10]. The encouraging results demonstrate that the IDP technique outperforms LBP and LDP in gender classification task, while it operates much faster than LDP.

The rest of this paper is organized as follows. Section 2 presents the proposed IDP method in detail. In section 3 , the experimental results are provided. The last section concludes the paper.

\section{High-ORDER DERIVATIVE PATTERN}

In this section, we propose a brief review of the conventional high-order local derivative pattern (LDP), then introduce the Interlaced Derivative Pattern (IDP) and discuss the superiority and efficiency of the new approach over LBP and LDP.

\section{A. Local Derivative Pattern}

The LDP operator is mainly based on capturing detailed relationships in a local neighborhood. While LBP encodes the binary results of the first-order derivative in the local neighborhood, the $\mathrm{n}^{\text {th }}$-order LDP can capture the changes of derivative directions among local neighbors. Therefore, more 
detailed discriminative features from the image will be obtained by LDP, which cannot be captured by LBP.

The original LDP was proposed for person identification by Zhang et al. [13]. The LDP operator finds the derivatives along four directions: $0^{\circ}, 45^{\circ}, 90^{\circ}$ and $135^{\circ}$. The $\mathrm{n}^{\text {th }}$-order directional LDP is defined as

$$
\begin{gathered}
L D P_{\alpha}^{n}\left(Z_{0}\right)=\left\{f \left(I_{\alpha}^{n-1}\left(Z_{0}\right), I_{\alpha}^{n-1}\left(Z_{0}\right),\right.\right. \\
f\left(I_{\alpha}^{n-1}\left(Z_{0}\right), I_{\alpha}^{n-1}\left(Z_{1}\right), \ldots, f\left(I_{\alpha}^{n-1}\left(Z_{0}\right), I_{\alpha}^{n-1}\left(Z_{8}\right)\right\}\right.
\end{gathered}
$$

where $Z_{0}$ is a point in the image $I(Z)$ and $Z_{i}, i=1, \ldots, 8$ is the neighboring point in a $3 \times 3$ neighborhood around $Z_{0}, I_{a}^{(n-1)}$ $\left(Z_{i}\right)$ is the $(\mathrm{n}-1)^{\text {th }}$-order derivative in $\alpha$ direction at $Z=Z_{i}$, and $f($,$) defined in (2) encodes the (\mathrm{n}-1)^{\mathrm{th}}$-order gradient transitions into binary patterns, providing an extra order pattern information on the local region.

$$
\begin{aligned}
& f\left(I_{\alpha}^{n-1}\left(Z_{0}\right), I_{\alpha}^{n-1}\left(Z_{i}\right)\right)= \\
& \left\{\begin{array}{ll}
0, & \text { if } I_{\alpha}^{n-1}\left(Z_{0}\right) \cdot I_{\alpha}^{n-1}\left(Z_{i}\right)>0 \\
1, & \text { if } I_{\alpha}^{n-1}\left(Z_{0}\right) \cdot I_{\alpha}^{n-1}\left(Z_{i}\right) \leq 0
\end{array}, i=1,2, \ldots, 8 .\right.
\end{aligned}
$$

The ${ }^{\text {th }}$-order LDP is defined as

$$
L D P^{n}(Z)=\left\{L D P_{\alpha}^{n}(Z) \mid \alpha=0^{\circ}, 45^{\circ}, 90^{\circ}, 135^{\circ}\right\}
$$

Therefore for each direction, LDP applies the encoding function on all neighbors, regardless the direction between the neighbor and the center pixel. Hence, it produces a 32-bit long representation for each pixel which makes the computational process much slow.

\section{B. Interlaced Derivative Pattern}

IDP is a fully directional derivative pattern that takes the advantage of more detailed high-order derivative descriptions and keeps the spatial relationships in local regions.

In this technique, an IDP image is produced for the original image. The IDP image is a four-channel derivative image, representing four directional $\mathrm{n}^{\text {th }}$-order derivative channels in $0^{\circ}, 45^{\circ}, 90^{\circ}$, and $135^{\circ}$, respectively. The order of derivatives is derived from the order of the IDP operator; i.e., for an $n^{\text {th }}$-order IDP operator, the IDP image with four $(\mathrm{n}-1)^{\mathrm{th}}$-order derivative channels is produced. These derivative channels present more detailed description of the image in all possible directions (see Fig. 1). A $3 \times 3$ neighborhood is selected around each point in the original image and the pixel is located in the IDP image. For each neighbor, the direction between the center and the neighbor is computed and the IDP image channel with the same direction is selected.

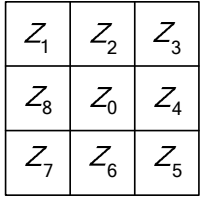

(a)

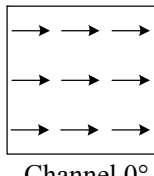

Channel $0^{\circ}$

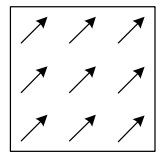

Channel $45^{\circ}$

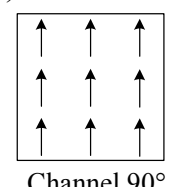

(b)

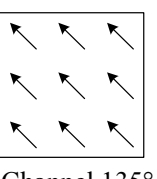

Channel $135^{\circ}$
Figure 1. (a) A $3 \times 3$ neighborhood around a pixel. (b) Four directional derivative channels in the IDP image.

The neighbor is thresholded with the center pixel value in the selected IDP channel and the result is encoded as a binary number. This thresholding actually encodes the binary result of the first-order derivative among local neighbors and produce an extra order for the IDP operator. The nth-order IDP operator is presented in (4).

$$
\begin{gathered}
I D P^{n}\left(Z_{0}\right)=\left\{f\left(I_{135}^{n-1}\left(z_{0}\right), I_{135}^{n-1}\left(z_{1}\right)\right), f\left(I_{90}^{n-1}\left(z_{0}\right), I_{90}^{n-1}\left(z_{2}\right)\right),\right. \\
f\left(I_{45}^{n-1}\left(z_{0}\right), I_{45}^{n-1}\left(z_{3}\right), f\left(I_{0}^{n-1}\left(z_{0}\right), I_{0}^{n-1}\left(z_{4}\right)\right),\right. \\
f\left(I_{15}^{n-1}\left(z_{0}\right), I_{135}^{n-1}\left(z_{5}\right)\right), f\left(I_{90}^{n-1}\left(z_{0}\right), I_{90}^{n-1}\left(z_{6}\right)\right), \\
\left.f\left(I_{45}^{n-1}\left(z_{0}\right), I_{45}^{n-1}\left(z_{7}\right)\right), f\left(I_{0}^{n-1}\left(z_{0}\right), I_{0}^{n-1}\left(z_{8}\right)\right)\right\}
\end{gathered}
$$

where the function $f$ is defined as

$$
f(x, y)= \begin{cases}1, & \text { if }(x-y) \geq 0 \\ 0, & \text { if }(x-y)<0\end{cases}
$$

Therefore in each direction, only the derivatives for the center point and its neighbor point in that particular direction will be calculated. This will dramatically decrease the length of the pixel representing code produced by the proposed operator compared to the LDP operator. LDP keeps the extra information in a local neighborhood, while the new approach encodes the relationships in the particular directions. In this way, IDP keeps only the more important information and makes the process much faster. It produces an 8-bit representation of each pixel, which makes the operator four times faster than LDP with a 32-bit representation of pixels. Also compared to LBP, IDP contains more detailed description by calculating the high-order derivative directional variations, while LBP provides first-order derivative information and is incapable of describing more detailed information. Fig. 2 illustrates the 2nd-order IDP operator and Fig. 3 shows the visualized results of the IDP operator on a sample image. 


\begin{tabular}{|l|l|l|l|l|}
\hline 9 & 2 & 5 & 3 & 8 \\
\hline 8 & 6 & 7 & 1 & 9 \\
\hline 2 & 1 & 4 & 3 & 6 \\
\hline 6 & 8 & 9 & 5 & 7 \\
\hline 5 & 4 & 3 & 8 & 1 \\
\multicolumn{5}{|c}{$Z_{0}$} \\
\multicolumn{5}{c}{}
\end{tabular}

(a)

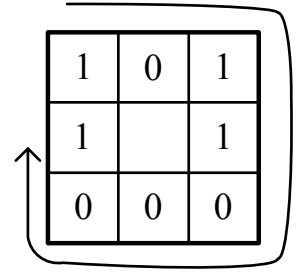

(c)

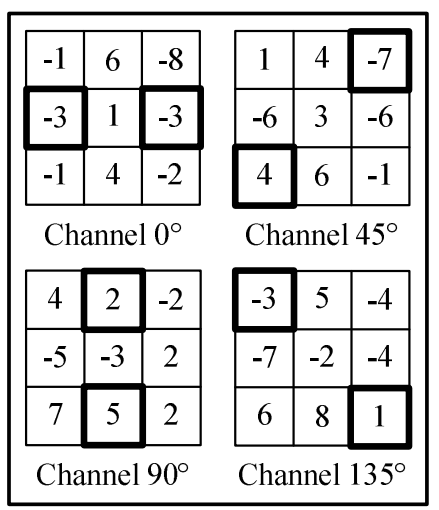

(b)

Binary number: 1011001
Figure 2. (a) $3 \times 3$ neighborhood in original image. (b) 4-channel IDP representation. (c) IDP code for 1 point.

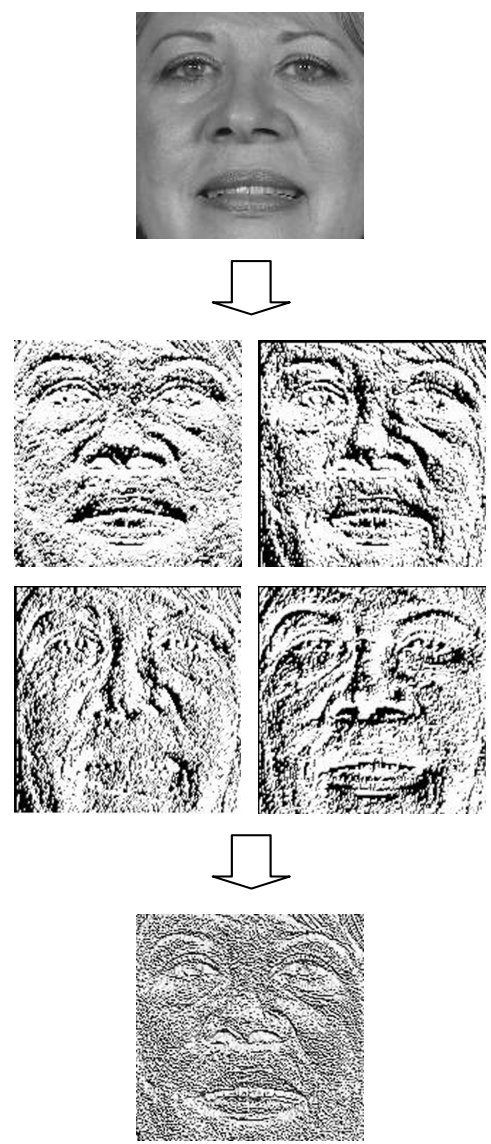

Figure 3. Visualized results of IDP code generation process for a face image

To extract the discriminative IDP features of the image, the image is divided into rectangular subregions represented by $\mathrm{Ri}, \ldots, \mathrm{RL}$, and the spatial histograms are used to model the distribution of Interlaced Derivative Patterns. Taking the spatial histograms of the subregions and concatenating them into an enhanced feature vector as the image descriptor is more robust against pose and illumination variations than the holistic methods [1].

\section{EXPERIMENTAL RESULTS AND DISCUSSION}

The FRGC ver.2.0 database [10] was used in our experiments. We selected 457 individuals; 262 male and 195 female subjects. All images were normalized with the two eyes and cropped to $160 \times 160$ images, so that each image contained little or no hair information.

In our experiments, we applied LDP and evaluated its feasibility on the gender classification for the first time. We also implemented the new IDP approach as an improvement to LDP technique and compared the performance and efficiency of IDP with LBP and LDP in gender recognition task. All three techniques were used to produce appropriate features for gender classification. In each case, the operator was applied on all images. The images were divided into $10 \times 10$ subregions and the image feature vector was produced by concatenating all subregion histograms.

The recognition rate was estimated with five-fold cross validation. A pair of mean male/female subjects was produced for each set of training subjects, and histogram intersection in (6) was applied to measure the similarity between the test subject and the mean male/female subjects.

$$
S_{H I}(H, S)=\sum_{i=1}^{B} \min \left(H_{i}, S_{i}\right)
$$

where $S_{H I}(H, S)$ is the histogram intersection statistic with $H=\left(H_{1}, \ldots, H_{8}\right)^{T}$ and $S=\left(S_{1}, \ldots, S_{8}\right)^{T}$.

Different orders for LDP and IDP operators were tested. We found that higher order of LDP is required for gender classification than for identification. The 4th-order LDP performed the best in gender recognition while the 3th-order had the best results in person identification [13]. For the proposed approach, the 2nd-order IDP had the highest performance and outperformed LBP and LDP.

Also different numbers of histogram bins in each subregion were experimented, and the recognition error rate curves of all the operators remained relatively flat (see Fig. 4).

Table I shows the error rates of the three operators for male and female subjects and demonstrates that IDP technique outperformed LBP and LDP techniques with the highest recognition rate of $91.2 \%$. The results show that the more detailed information extracted by IDP and LDP is more effective for gender classification than the first-order derivative information of LBP.

Table II demonstrates the average computation time for code generation and matching process for a sample image based on Pentium $4(2.8 \mathrm{GHz})$ implemented in MATLAB R2008a. As shown, the proposed approach performs over four times faster than LDP. 


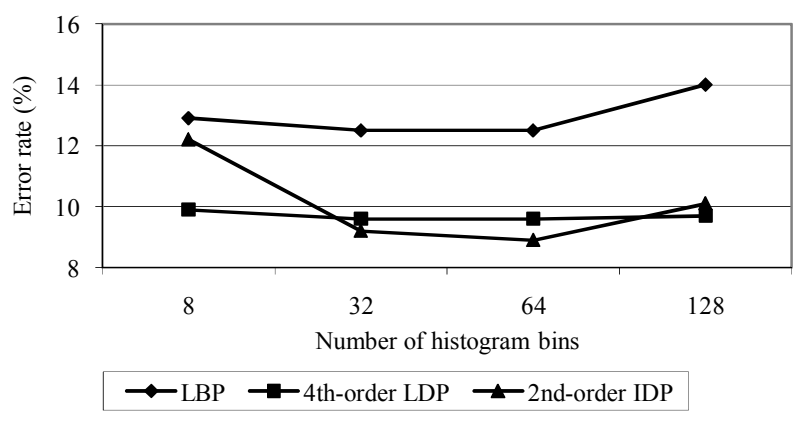

Figure 4. Classification error rates

TABLE I. CLASSIFICATION ERROR RATES OF DIFFERENT TECHNIQUES WITH 64 HISTOGRAM BINS

\begin{tabular}{|c|c|c|c|}
\hline \multirow{2}{*}{ Method } & \multicolumn{3}{|c|}{ error rate } \\
\cline { 2 - 4 } & Female & Male & Overall \\
\hline LBP & $10.8 \%$ & $13.7 \%$ & $12.5 \%$ \\
\hline $4^{\text {th }}$-order LDP & $14.9 \%$ & $5.7 \%$ & $9.6 \%$ \\
\hline $2^{\text {nd }}$-order IDP & $\mathbf{1 3 . 8 \%}$ & $\mathbf{5 \%}$ & $\mathbf{8 . 8} \%$ \\
\hline
\end{tabular}

TABLE II. AVERAGE COMPUTATION TIME

\begin{tabular}{|c|c|c|c|c|c|}
\hline & LBP & LDP $^{2}$ & LDP $^{3}$ & LDP $^{4}$ & IDP \\
\hline Time(sec) & 0.4 & 2.1 & 2.2 & 2.1 & $\mathbf{0 . 4}$ \\
\hline
\end{tabular}

For each direction, LDP applies the encoding function on all neighbors around a pixel, while IDP encodes the relationship between the pixel and its neighbor in that particular direction. Therefore compared to LDP, our approach contains less noise and redundancy and produces compact representative features. On the other hand, although increasing the order of the operator improves the accuracy by extracting more detailed information, it amplifies the noise which defects the results accuracy. Therefore in higher orders, the recognition accuracy start to decline and a 'best-result' order is determined for the operators.

\section{CONCLUSION}

In this paper, we present a high-order directional texture representation, the Interlaced Derivative Pattern (IDP), for gender classification. It contains more discriminating information than the first-order features (LBP) and dramatically reduces the computational complexity compared with the conventional high-order features (LDP). The feasibility and efficiency of the proposed approach is evaluated on FRGC ver.2.0 database and compared against LBP and LDP methods. The promising results of the proposed approach in gender classification imply its potential capability for other face recognition tasks. Besides, the best-result order drops down from the fourth in LDP to the second in IDP, which is an interesting issue for looking into detail in further study.

\section{REFERENCES}

[1] T. Ahonen, A. Hadid, and M. Pietikainen, "Face Description with Local Binary Patterns: Application to Face Recognition," IEEE Trans. on Pattern Analysis and Machine Intelligence, vol. 28(12), 2006, pp. 2037-2041.

[2] R. Brunelli and T. Poggio, "Hyperbf Networks for Gender Classification," DARPA Image Understanding Workshop, 1992, pp. 311-314.

[3] G. Cottrell and J. Metcalfe, "Empath: Face, Emotion, and Gender Recognition Using Holons," Neural Information Processing Systems, vol. 3, 1991, pp. 564-571.

[4] B. Golomb, D. Lawrence, and T. Sejnowski, "Sexnet: A Neural Network Identifies Sex from Human Faces," Advances in neural information processing systems, vol. 3, 1991, pp. 572-577.

[5] S. Gutta and H. Wechsler, "Gender Classification of Human Faces Using Hybrid Classifier Systems," International Conference on Neural Networks, vol. 3, 1997, pp. 1353-1358.

[6] H.C. Lian and B.L. Lu, "Multi-View Gender Classification Using Multi-Resolution Local Binary Patterns and Support Vector Machines," International Journal of Neural Systems, vol. 17(6), 2007, pp. 479-487.

[7] E. Makinen and R. Raisamo, "An Experimental Comparison of Gender Classification Methods," Pattern Recognition Letters, vol. 29(10), 2008, pp. 1544-1556.

[8] B. Moghaddam and Y. Ming-Hsuan, "Learning Gender with Support Faces," IEEE Trans. on Pattern Analysis and Machine Intelligence, vol. 24(5), 2002, pp. 707-711.

[9] T. Ojala, M. Pietikainen, and T. Maenpaa, "Multiresolution Gray-Scale and Rotation Invariant Texture Classification with Local Binary Patterns," IEEE Trans. on Pattern Analysis and Machine Intelligence, vol. 24(7), 2002, pp. 971-987.

[10] P.J. Phillips, P.J. Flynn, T. Scruggs, K.W. Bowyer, C. Jin, K. Hoffman, J. Marques, M. Jaesik, and W. Worek, "Overview of the Face Recognition Grand Challenge," IEEE Computer Society Conference on Computer Vision and Pattern Recognition, vol. 1, 2005, pp. 947-954.

[11] Y. Saatci and C. Town, "Cascaded Classification of Gender and Facial Expression Using Active Appearance Models," 7th International Conference on Automatic Face and Gesture Recognition, 2006, pp. 393-398.

[12] Z. Sun, G. Bebis, X. Yuan, and S.J. Louis, "Genetic Feature Subset Selection for Gender Classification: A Comparison Study," Sixth IEEE Workshop on Applications of Computer Vision, 2002, pp. 165-170.

[13] B. Zhang, Y. Gao, S. Zhao, and J. Liu, "Local Derivative Pattern Versus Local Binary Pattern: Face Recognition with High-Order Local Pattern Descriptor," IEEE Transactions on Image Processing, vol. 19(2), 2010, pp. 533-544. 